\title{
THE KOBAYASHI AND BERGMAN METRICS ON GENERALIZED THULLEN DOMAINS
}

\author{
K. T. HAHN AND P. PFLUG
}

(Communicated by Irwin Kra)

\begin{abstract}
A comparison theorem of the Kobayashi metric and the Bergman metric is obtained on generalized Thullen domains in $\mathbf{C}^{2}$. This theorem is then used to obtain a lower estimate for the Kobayashi metric. It is noted that the lower estimate obtained is best possible.
\end{abstract}

1. Introduction. In this note we obtain lower estimates for the Kobayashi metric on generalized Thullen domains.

We call

$$
D_{p q}=\left\{z \in \mathbf{Z}^{2}:\left|z_{1}\right|^{2 / p}+\left|z_{2}\right|^{2 / q}<1\right\}
$$

a generalized Thullen domain of type $(p, q)$ if $p, q>0$, and prove a comparison theorem (Theorem 1) for the Kobayashi and Bergman metrics on $D_{p q}$. Using this result we obtain the lower estimates for the Kobayashi metric.

More precisely, we prove the following

THEOREM. Let $C_{D_{p q}}, K_{D_{p q}}$ and $B_{D_{p q}}$ denote the Carathéodory, Kobayashi and Bergman metric of $D_{p q}$, respectively.

(a) For each $z \in D_{p q}$ and $X \in \mathbf{C}^{2}$, and $p, q>0$, there exist positive constants $C_{j}(j=1,2,3)$, depending only on $p$ and $q$, such that

$$
\frac{C_{1}|X|}{\left|r_{p q}(z)\right|^{N(p, q)}} \leq C_{2} B_{D_{p q}}(z, X) \leq K_{D_{p q}}(z, X) \leq \frac{C_{3}|X|}{d_{D_{p q}}(z)}
$$

where $d_{D_{p q}}(z)$ denotes the euclidean boundary distance of $z \in D_{p q}, N(p, q)=$ $\frac{1}{2} \min (1, p, q)$ and $r_{p q}(z)=\left|z_{1}\right|^{2 / p}+\left|z_{2}\right|^{2 / q}-1$.

(b) For the values of $p$ and $q$ so that $D_{p q}$ is convex, we obtain for $z \in D_{p q}$ and $X \in \mathbf{C}^{2}$

$$
\begin{aligned}
\frac{C_{1}|X|}{\left|r_{p q}(z)\right|^{N(p, q)}} & \leq C_{2} B_{D_{p q}}(z, X) \leq K_{D_{p q}}(z, X) \\
& =C_{D_{p q}}(z, X) \leq B_{D_{p q}}(z, X) \leq \frac{C_{3}|X|}{\left|r_{p q}(z)\right|}
\end{aligned}
$$

where $C_{j}(j=1,2,3)$ are positive constants.

REMARK. $D_{p q}$ is convex if and only if $0<p, q \leq 2$.

In the case where $D_{p q}$ has real analytic boundary (when $1 / p, 1 / q \in \mathrm{N}$ ), similar results have already been obtained in $[3,5,6, \mathbf{1 4}]$. We note here that the estimates

Received by the editors April 22, 1987 and, in revised form, August 31, 1987.

1980 Mathematics Subject Classification (1985 Revision). Primary 32H15.

Research of the first author partially supported by a 1986/1987 Fulbright Research Grant at Universität Osnabrück-Abteilung Vechta. 
obtained in the theorem include weakly pseudoconvex domains with nonsmooth boundaries. The lower estimates obtained in the theorem is then applied to obtain the Hölder continuity of proper holomorphic mappings $F: D \rightarrow D_{1 p}$ for $p<2$ when $D$ is a bounded pseudoconvex domain with $\mathbf{C}^{2}$-boundary.

2. Comparison theorem. In this paragraph we prove a comparison theorem of the Kobayashi metric and the Bergman metric for $D_{p q}$.

First we need the following lemmas:

LEMMA 1. Every boundary point of $D_{p q}$ is a peak point with respect to $A\left(D_{p q}\right)$, where $A\left(D_{p q}\right)$ is the space of functions holomorphic in $D_{p q}$ and continuous in the closure $\bar{D}_{p q}$.

PROOF. Let $z^{0}=\left(z_{1}^{0}, z_{2}^{0}\right)$ be a boundary point of $D_{p q}$. If $z_{1}^{0}=0, f(z)=$ $\left(z_{2}^{0}+z_{2}\right) / 2$ is a peak function at $z^{0}$. Similarly, $g(z)=\left(z_{1}^{0}+z_{1}\right) / 2$ is a peak function at $z^{0}$ when $z_{2}^{0}=0$. If $z_{1}^{0} \neq 0 \neq z_{2}^{0}$, then $h(z)=\frac{1}{2}\left[1+\left(\overline{z_{1}^{0}} z_{1}\right)^{1 / p}+\left(\overline{z_{2}^{0}} z_{2}\right)^{1 / q}\right]$ provides a local peak function at $z^{0}$. Since $\bar{D}_{p q}$ has a Stein neighborhood basis, by Rossi's local peak point theorem [8], it follows that $z^{0}$ is a peak point with respect to $A\left(D_{p q}\right)$.

LEMMA 2. Let $z^{0} \in \partial D_{p q}$ and let $V=V\left(z^{0}\right)$ be its neighborhood. Then there exists a neighborhood $V^{\prime}=V^{\prime}\left(z^{0}\right) \subset \subset V$ and a constant $C>0$ such that for $z \in D_{p q} \cap V^{\prime}$ and $X \in \mathbf{C}^{2}$, we have

$$
K_{D_{p q} \cap V}(z, X) \leq C K_{D_{p q}}(z, X) .
$$

PROOF. According to Lemma 4 of [7], for all $z \in D_{p q} \cap V$ and $X \in \mathbf{C}^{2}$,

$$
K_{D_{p q} \cap V}(z, X) \leq \operatorname{coth} D^{*}(z) K_{D_{p q}}(z, X),
$$

where $D^{*}(z)=\inf \left\{D_{D_{p q}}^{*}(z, w): w \in D_{p q} \backslash V\right\}$,

$$
D_{D_{p q}}^{*}(z, w)=\inf \left\{\rho(a, b): \exists f: \Delta \rightarrow D_{p q} \text { such that } f(a)=z \text { and } f(b)=w\right\}
$$

and $\rho$ is the Poincare distance on the unit disc $\Delta \subset \mathbf{C}$.

Therefore, what remains is to estimate $\operatorname{coth} D^{*}(z)$ from below. Since coth is a monotonically decreasing function, we need a uniform lower estimate for $D^{*}(z)$.

Let $f$ be a peak function at $z^{0}$ with respect to $A\left(D_{p q}\right)$ and set

$$
\eta=\sup \left\{|f(\varsigma)|: \varsigma \in \bar{D}_{p q} \backslash V\right\}<1 \text {. }
$$

Choose a neighborhood $V^{\prime}=V^{\prime}\left(z^{0}\right)$ of $z^{0}$ such that for all $\varsigma \in D_{p q} \cap V^{\prime},|f(\varsigma)| \geq$ $\eta^{\prime}=(1+\eta) / 2$. So, for all $z \in D_{p q} \cap V^{\prime}$ and $w \in D_{p q} \backslash V$ we have

$$
D_{D_{p q}}^{*}(z, w) \geq k_{D_{p q}}(z, w) \geq c_{D_{p q}}(z, w) \geq \rho(f(z), f(w)) \geq \rho\left(\eta, \eta^{\prime}\right)>0,
$$

where $k$ and $c$ denote the Kobayashi and Carathéodory distances, respectively. This implies: $D^{*}(z) \geq C=\tanh ^{-1}(1 /(\eta+2))>0$ and proves the lemma.

THEOREM 1. There exists a constant $C_{p q}>0$ such that

$$
B_{D_{p q}}(z, X) \leq C_{p q} K_{D_{p q}}(z, X)
$$

for each $z \in D_{p q}$ and $X \in \mathbf{C}^{2}$.

ProOF. First we observe that if $p=1$ and $q>0$, then the holomorphic sectional curvature of the Bergman metric of $D_{1 q}$ is bounded from above by a 
negative constant $[\mathbf{1}, \mathbf{2}]$. Therefore, by $[10]$ there exists a constant $C_{1 q}>0$ such that

$$
B_{D_{1 q}}(z, X) \leq C_{1 q} K_{D_{1 q}}(z, X)
$$

for all $z \in D_{1 q}$ and $X \in \mathbf{C}^{2}$.

Suppose there are sequences $\left\{z^{\nu}\right\}$ in $D_{p q}$ with $z^{\nu} \rightarrow z^{0}=\left(z_{1}^{0}, z_{2}^{0}\right) \in \partial D_{p q}$ and $\left\{X^{\nu}\right\}$ in $\mathbf{C}^{2}$ with $\left|X^{\nu}\right|=1$ and $X^{\nu} \rightarrow X \in \mathbf{C}^{2}$ such that

$$
B_{D_{p q}}\left(z^{\nu}, X^{\nu}\right) / K_{D_{p q}}\left(z^{\nu}, X^{\nu}\right) \rightarrow \infty \text {. }
$$

We may assume $z_{1}^{0} \neq 0$. Let $\Phi: D_{1 q} \rightarrow D_{p q}$ be the map defined by

$$
z=\Phi\left(\varsigma_{1}, \varsigma_{2}\right)=\left(\varsigma_{1}^{p}, \varsigma_{2}\right) \text {. }
$$

Then $\left|z_{1}\right|^{2 / p}+\left|z_{2}\right|^{2 / q}=\left|\zeta_{1}\right|^{2}+\left|\zeta_{2}\right|^{2 / q}<1$ for all $\zeta \in D_{1 q}$. Let

$$
\begin{aligned}
& U=\left\{\left(\varsigma_{1}, \varsigma_{2}\right) \in \mathbf{C}^{2}:\left|\arg \zeta_{1}-\arg \varsigma_{1}^{0}\right|<\pi / p, \varsigma_{2} \in \mathbf{C}\right\}, \\
& V=\left\{\left(z_{1}, z_{2}\right) \in \mathbf{C}^{2}:\left|\arg z_{1}-\arg z_{1}^{0}\right|<\pi, z_{2} \in \mathbf{C}\right\} .
\end{aligned}
$$

Then $\Phi$ is a biholomorphic map of $D_{1 q} \cap U$ onto $D_{p q} \cap V$. Let $V^{\prime}$ be a neighborhood of $z^{0}$, chosen according to Lemma 2 and let $U^{\prime}=\Phi^{-1}\left(V^{\prime}\right)$. By the invariant property of $K$ and $B$,

$$
\left(B_{D_{p q} \cap V} / K_{D_{p q} \cap V}\right)\left(z^{\nu}, X^{\nu}\right)=\left(B_{D_{1 q} \cap U} / K_{D_{1 q} \cap U}\right)\left(\varsigma^{\nu}, Y^{\nu}\right)
$$

for sufficiently large $\nu$ where $z^{\nu}=\Phi\left(\varsigma^{\nu}\right)$ and $X^{\nu}=d \Phi\left(z^{\nu}\right) Y^{\nu}$. By Lemma 2, there exists a constant $C_{1}>0$ such that

$$
K_{D_{p q} \cap V}(z, X) \leq C_{1} K_{D_{p q}}(z, X)
$$

for all $z \in D_{p q} \cap V^{\prime}$ and $X \in \mathbf{C}^{2}$. According to [6], there exists a constant $C_{2}=C_{2}\left(U, U^{\prime}\right)>0$ such that

$$
\left(B_{D_{1 q} \cap U} / B_{D_{1 q}}\right)\left(\varsigma^{\nu}, Y^{\nu}\right) \leq C_{2}
$$

for sufficiently large $\nu$. Therefore, by (7), (6), (8) and (4), we obtain

$$
\begin{aligned}
{\left[\frac{B_{D_{p q}}}{C_{1} K_{D_{p q}}}\right]\left(z^{\nu}, X^{\nu}\right) } & \leq\left[\frac{B_{D_{p q} \cap V}}{K_{D_{p q} \cap V}}\right]\left(z^{\nu}, X^{\nu}\right) \\
& =\left[\frac{B_{D_{1 q} \cap U}}{K_{D_{1 q} \cap U}}\right]\left(\varsigma^{\nu}, Y^{\nu}\right) \leq C_{2}\left[\frac{B_{D_{1 q}}}{K_{D_{1 q}}}\right]\left(\varsigma^{\nu}, Y^{\nu}\right) \leq C_{2} C_{1 q},
\end{aligned}
$$

for sufficiently large $\nu$ which contradicts (5).

Let $B_{2}^{*}=\left\{z \in \mathbf{C}^{2}: N^{*}(z)<1\right\}, N^{*}(z)^{2}=\frac{1}{2}\left(\left|z_{1}\right|^{2}+\left|z_{2}\right|^{2}+\left|z_{1}^{2}+z_{2}^{2}\right|\right)$, the maximal ball given in [9]. Due to the convexity of $B_{2}^{*}$ and a result of [12], we obtain the following

COROLLARY. There exists a constant $C>0$ such that

$$
K_{B_{2}^{*}}(z, X) \leq B_{B_{2}^{*}}(z, X) \leq C K_{B_{2}^{*}}(z, X)
$$

for $z \in B_{2}^{*}$ and $X \in \mathbf{C}^{2}$.

REMARK. It would be interesting to know whether for all bounded pseudoconvex Reinhardt domains in $\mathbf{C}^{2}$ containing the origin the comparison result $K \geq C B$ remains true. 
3. Estimates of the Bergman metric on $D_{p q}$. We consider first the Thullen domain:

$$
D_{p}=D_{1 p}=\left\{z \in \mathbf{C}^{2}:\left|z_{1}\right|^{2}+\left|z_{2}\right|^{2 / p}<1\right\}, \quad p>0 .
$$

The Bergman kernel of $D_{p}$ is well known and given by

$$
K(z)=\frac{D(z)}{\pi^{2} C(z)^{3}}\left(1-\left|z_{1}\right|^{2}\right)^{p-2}, \quad z \in D_{p}
$$

where

$$
\begin{aligned}
& C(z)=\left(1-\left|z_{1}\right|^{2}\right)^{p}-\left|z_{2}\right|^{2}, \\
& D(z)=(p+1)\left(1-\left|z_{1}\right|^{2}\right)^{p}+(p-1)\left|z_{2}\right|^{2} .
\end{aligned}
$$

See [4].

A straightforward computation yields the Bergman metric in the direction $X \in$ $\mathrm{C}^{2}$ at $z \in D_{p}$ as follows:

$$
B_{D_{p}}^{2}(z, X)=\sum_{\alpha, \beta=1}^{2} T_{\alpha \bar{\beta}} X_{\alpha} \bar{X}_{\beta},
$$

where $T_{\alpha \bar{\beta}}(z)=\left(\partial^{2} / \partial z_{\alpha} \partial \bar{z}_{\beta}\right) \log K(z), \alpha, \beta=1,2$, and

$$
\begin{aligned}
T_{1 \overline{1}}(z)= & \frac{2-p}{\left(1-\left|z_{1}\right|^{2}\right)^{p}}+\frac{3 p\left[C(z)+p\left|z_{1}\right|^{2}\left|z_{2}\right|^{2}\right]\left(1-\left|z_{1}\right|^{2}\right)^{p-2}}{C^{2}(z)} \\
& -p(p+1) \frac{\left[D(z)-p(p-1)\left|z_{1}\right|^{2}\left|z_{2}\right|^{2}\right]\left(1-\left|z_{1}\right|^{2}\right)^{p-2}}{D^{2}(z)} \\
T_{1 \overline{2}}(z)= & \bar{T}_{2 \overline{1}}(z)=p \bar{z}_{1} z_{2}\left(1-\left|z_{1}\right|^{2}\right)^{p-1}\left(\frac{1}{C^{2}(z)}+\frac{p^{2}-1}{D^{2}(z)}\right) \\
T_{2 \overline{2}}(z)= & \left(1-\left|z_{1}\right|^{2}\right)^{p}\left(\frac{1}{C^{2}(z)}+\frac{1}{D^{2}(z)}\right)
\end{aligned}
$$

Define

$$
T(z)=\operatorname{det}\left(T_{\alpha \bar{\beta}}(z)\right)=T_{1 \overline{1}} T_{2 \overline{2}}(z)-\left|T_{1 \overline{2}}(z)\right|^{2} .
$$

LEMMA 3. There are positive constants $a_{1}$ and $a_{2}$, depending only on $p$, such that

$$
a_{1} K(z) \leq T(z) \leq a_{2} K(z)
$$

for all $z \in D_{p}$ and $p>0$.

PROOF. Set

$$
t(z)=\left|z_{1}\right| \quad \text { and } \quad \varepsilon(z)=\frac{\left|z_{2}\right|^{2}}{\left(1-\left|z_{1}\right|^{2}\right)^{p}}
$$

Then

$$
\left|z_{2}\right|^{2}=\left(1-|t(z)|^{2}\right)^{p} \varepsilon(z) \quad \text { and } \quad 0 \leq \varepsilon(z)<1 \quad \text { for } z \in D_{p} .
$$

By a formal computation, we obtain on $D_{p}$,

$$
\frac{b_{1}}{\left(1-t^{2}\right)^{p+2}(1-\varepsilon)^{3}} \leq T \leq \frac{b_{2}}{\left(1-t^{2}\right)^{p+2}(1-\varepsilon)^{3}}
$$


where $b_{1}$ and $b_{2}$ are positive constants depending only on $p$. Since

$$
K=\frac{p+1+(p-1) \varepsilon}{\pi^{2}\left(1-t^{2}\right)^{p+2}(1-\varepsilon)^{3}},
$$

the quotient $T / K$ is bounded by $a_{1}$ and $a_{2}$ as given in (3).

LEMMA 4. For each $z \in D_{p}$ and $X \in \mathbf{C}^{2}$,

$$
\frac{T(z)}{T_{1 \overline{1}}(z)+T_{2 \overline{2}}(z)}|X|^{2} \leq B_{D_{p}}^{2}(z, X) \leq\left(T_{1 \overline{1}}(z)+T_{2 \overline{2}}(z)\right)|X|^{2} .
$$

ProOF. Since $B_{D_{p}}^{2}(z, X)$ is a positive definite form, it can be written as

$$
\lambda_{1}(z)|X|^{2} \leq B_{D_{p}}^{2}(z, X) \leq \lambda_{2}(z)|X|^{2}
$$

here $\lambda_{1}$ and $\lambda_{2}$ are the eigenvalues of the characteristic equation

$$
\operatorname{det}\left(T_{\alpha \bar{\beta}}-\lambda I\right)=0
$$

and given by

$$
\left\{\begin{array}{l}
\lambda_{2} \\
\lambda_{1}
\end{array}\right\}=\frac{1}{2}\left(T_{1 \overline{1}}+T_{2 \overline{2}}\right)\left\{1 \pm \sqrt{1-\frac{4 T}{\left(T_{1 \overline{1}}+T_{2 \overline{2}}\right)^{2}}}\right\} .
$$

Clearly, $\lambda_{2} \leq T_{1 \overline{1}}+T_{2 \overline{2}}$ and $\lambda_{1} \geq T /\left(T_{1 \overline{1}}+T_{2 \overline{2}}\right)$. The latter inequality follows from the following observation:

$$
1-\sqrt{1-a}=\frac{a}{1+\sqrt{1-a}} \geq \frac{a}{2} \quad \text { if } 0 \leq a<1 .
$$

LEMMA 5. There exists a positive constant d, depending only on $p$, such that on $D_{p}$,

$$
T_{1 \overline{1}}+T_{2 \overline{2}} \leq \begin{cases}\frac{d}{\left(1-t^{2}\right)^{2}(1-\varepsilon)^{2}}, & 0<p \leq 2, \\ \frac{d}{\left(1-t^{2}\right)^{p}(1-\varepsilon)^{2}}, & 2<p .\end{cases}
$$

PROOF. It follows from (2b) when we express $T_{1 \overline{1}}$ and $T_{2 \overline{2}}$ in terms of $t$ and $\varepsilon$ and sum them up.

LEMMA 6. There exists a positive constant $C$ depending on $p$ such that on $D_{p}$,

$$
\frac{T}{T_{1 \overline{1}}+T_{2 \overline{2}}} \geq \begin{cases}\frac{C}{\left(1-t^{2}\right)^{p}(1-\varepsilon)}, & 0<p \leq 2, \\ \frac{C}{\left(1-t^{2}\right)^{2}(1-\varepsilon)}, & 2<p .\end{cases}
$$

PROOF. It follows from Lemma 3 and Lemma 5.

Combining Lemma 5 and Lemma 6 together with Lemma 4, we get

LEMMA 7. For each $z \in D_{p}$ and $X \in \mathbf{C}^{2}$, there exist positive constants $C$ and $d$ depending on $p$ such that

$$
\begin{array}{ll}
\frac{C|X|^{2}}{\left(1-t^{2}(z)\right)^{p}(1-\varepsilon(z))} \leq B_{D_{p}}^{2}(z, X) \leq \frac{d|X|^{2}}{\left(1-t^{2}(z)\right)^{2}(1-\varepsilon(z))^{2}}, & 0<p \leq 2, \\
\frac{C|X|^{2}}{\left(1-t^{2}(z)\right)^{2}(1-\varepsilon(z))} \leq B_{D_{p}}^{2}(z, X) \leq \frac{d|X|^{2}}{\left(1-t^{2}(z)\right)^{p}(1-\varepsilon(z))^{2}}, & 2<p .
\end{array}
$$


THEOREM 2. Let $r_{p}(z)=\left|z_{1}\right|^{2}+\left|z_{2}\right|^{2 / p}-1$ be the defining function for $D_{p}$. Then for each $z \in D_{p}$ and $X \in \mathbf{C}^{2}$ there exist positive constants $a$ and $b$ depending on $p$ such that

$$
\begin{aligned}
& \frac{b|X|^{2}}{\left|r_{p}(z)\right|^{2}} \geq B_{D_{p}}^{2}(z, X) \geq \begin{cases}\frac{a|X|^{2}}{\left|r_{p}(z)\right|^{p}}, & 0<p \leq 1, \\
\frac{a|X|^{2}}{\left|r_{p}(z)\right|\left(1-\left|z_{1}\right|^{2}\right)^{p-1}}, & 1<p \leq 2,\end{cases} \\
& \frac{b|X|^{2}}{\left|r_{p}(z)\right|^{2}\left(1-\left|z_{1}\right|^{2}\right)^{p-2}} \geq B_{D_{p}}^{2}(z, X) \geq \frac{a|X|^{2}}{\left|r_{p}(z)\right|\left(1-\left|z_{1}\right|^{2}\right)}, \quad 2<p .
\end{aligned}
$$

In particular, if $0<p<2$, then we have

$$
\frac{\tilde{b}|X|^{2}}{d_{D_{p}}(z)^{2}} \geq B_{D_{p}}^{2}(z, X) \geq \frac{\tilde{a}|X|^{2}}{d_{D_{p}}(z)^{\tilde{p}}}
$$

where $\tilde{a}$ and $\tilde{b}$ are some positive constants depending on $p$ and $\tilde{p}=\min (p, 1)$.

PROOF. Theorem 2 follows from Lemma 7 when we observe that

$$
\left|r_{p}(z)\right|=\left(1-\left|z_{1}\right|^{2}\right)\left(1-\varepsilon(z)^{1 / p}\right) .
$$

If $0<p<2$, then $D_{p}$ is a convex domain with $C^{1}$-boundary. Therefore, by a result of $[12]$ and Theorem 2 , we have

COROLlaRY 1. If $0<p<2$, then there exist positive constants $a$ and $b$ depending only on $p$ such that for all $z \in D_{p}$ and $X \in \mathbf{C}^{2}$,

$\frac{a|X|}{d_{D_{p}}(z)^{\tilde{p} / 2}} \leq C_{D_{p}}(z, X)=K_{D_{p}}(z, X) \leq B_{D_{p}}(z, X) \leq \frac{b|X|}{d_{D_{p}}(z)} \quad$ with $\tilde{p}=\min (p, 1)$.

Following [14] and using an extension of Hopf's lemma [13, Theorem $3^{\prime}$, we obtain

COROLlaRY 2. Let $D \subset \mathrm{C}^{2}$ be a bounded pseudoconvex domain with $C^{2}$ boundary. If $F: D \rightarrow D_{p}(0<p<2)$ is a proper holomorphic map, then $F$ is Hölder continuous of some order $\alpha>0$ in $D$, where $\alpha$ is independent of $F$.

REMARK. In the case of $1 / p \in \mathbf{N}$, the above inequality was already obtained in [14]. Various lower estimates for invariant metrics have been obtained by many authors $[\mathbf{3}, \mathbf{5}, \mathbf{6}]$ in the context of proper holomorphic mappings on domains with real analytic boundaries.

THEOREM 3. There exist positive constants $C_{1}$ and $C_{2}$ depending only on $p$ and $q$ such that for all $z \in D_{p q}$ and $X \in \mathbf{C}^{2}$,

$$
\frac{C_{1}|X|}{\left|r_{p q}(z)\right|^{N(p, q)}} \leq B_{D_{p q}}(z, X) \leq \frac{C_{2}|X|}{\left|r_{p q}(z)\right|\left(1-\|\left. z\right|^{m(p, q)}\right)^{M(p, q)-1}},
$$

where $N(p, q)=\frac{1}{2} \min (1, p, q), M(p, q)=1+\frac{1}{2} \max (0, p-2, q-2), m(p, q)=$ $2 / \max (p, q)$ and $\|z\|=\max \left(\left|z_{1}\right|,\left|z_{2}\right|\right)$.

PROOF. It follows from Theorem 2 when we use the localization technique of the Bergman metric as in the proof of Theorem 1 and the standard compactness argument.

In view of Theorem 1 we have the following corollary. 
COROLlaRY. There exist positive constants $C_{j}(j=1,2,3)$ depending only on $p$ and $q$ such that for all $z \in D_{p q}$ and $X \in \mathbf{C}^{2}$,

$$
\frac{C_{1}|X|}{\left|r_{p q}(z)\right|^{N(p, q)}} \leq C_{2} B_{D_{p q}}(z, X) \leq K_{D_{p q}}(z, X) \leq \frac{C_{3}|X|}{d_{D_{p q}}(z)}
$$

for all $p, q>0$. For the values of $p$ and $q$ for which $D_{p q}$ is convex, we have for all $z \in D_{p q}$ and $X \in \mathbf{C}^{2}$,

$$
\begin{aligned}
\frac{C_{1}|X|}{\left|r_{p q}(z)\right|^{N(p, q)}} & \leq C_{2} B_{D_{p q}}(z, X) \leq K_{D_{p q}}(z, X)=C_{D_{p q}}(z, X) \\
& \leq B_{D_{p q}}(z, X) \leq \frac{C_{3}|X|}{\left|r_{p q}(z)\right|}
\end{aligned}
$$

where $N(p, q), m(p, q)$ and $\|z\|$ are given as in Theorem 3 .

4. Concluding remarks. In this paragraph we will discuss the special case $D_{22}$ to show that the order of the asymptotic boundary growth of the Kobayashi metric obtained in $\S 3$ is sharp. Using the biholomorphic map $F: D_{22} \rightarrow B_{2}^{*}$ with $F\left(z_{1}, z_{2}\right)=\left(z_{1}+z_{2}, i\left(z_{2}-z_{1}\right)\right)$ and $B_{2}^{*}=\left\{z \in \mathbf{C}^{2}: \frac{1}{2}\left|z_{1}+i z_{2}\right|+\frac{1}{2}\left|z_{1}-i z_{2}\right|<1\right\} \subset$ $\Delta^{2}$, we obtain the inequality

$$
K_{D_{22}}(z, X) \geq \max \left(\frac{\left|X_{1}+X_{2}\right|}{1-\left|z_{1}+z_{2}\right|}, \frac{\left|X_{1}-X_{2}\right|}{1-\left|z_{1}-z_{2}\right|}\right) .
$$

Therefore along the curve $z(t)=(t,(1-t) \varepsilon(t)), 0 \leq t<1,0 \leq \varepsilon(t)<1$, we have

$$
K_{D_{22}}(z, X) \geq C|X| /\left|r_{22}(z)\right|
$$

for some constant $C>0$ if $\left|X_{1}+X_{2}\right| \geq \varepsilon_{0}|X|, \varepsilon_{0}>0$. This shows that there are directions in which we can get better lower estimates. On the other hand, using the map

$$
\Phi: E_{t}:=\left\{\lambda \in \mathbf{C}:\left|\lambda+\frac{t+(1-t) \varepsilon(t)}{2}\right|+\left|\lambda-\frac{t+(1-t) \varepsilon(t)}{2}\right|<1\right\} \rightarrow D_{22},
$$

given by

$$
\Phi(\lambda):=\left(\lambda+\frac{t+(1-t) \varepsilon(t)}{2},-\lambda+\frac{t+(1-t) \varepsilon(t)}{2}\right)
$$

we obtain the upper estimate

$$
K_{D_{22}}(z(t),(1,-1)) \leq \frac{C|(1,-1)|}{(1-t) \sqrt{1-\varepsilon(t)}}=\frac{C^{\prime}}{\left|r_{22}(z(t))\right|^{1 / 2}(1-t)^{1 / 2}},
$$

thus confirming the order we obtained in $\S 3$ is sharp in this case.

It should be pointed out that the domain $D_{22}$ may be of some independent interests, due to the fact that it is biholomorphically equivalent to the maximal ball $B_{2}^{*}$ [9]. As it is well known [11] $\operatorname{Aut}\left(D_{22}\right)$ is highly nontransitive. In fact, it consists only of rotations. Thus it seems to be difficult to find a closed form of its Kobayashi metric. But using Bell's transformation formula for proper holomorphic mappings we obtain the Bergman kernel function $K$ of $D_{22}$ :

$$
K(z, \bar{w})=\frac{2}{\pi^{2}} \frac{3(1-\langle z, w\rangle)^{2}(1+\langle z, w\rangle)+4 z_{1} z_{2} \bar{w}_{1} \bar{w}_{2}(5-3\langle z, w\rangle)}{\left[(1-\langle z, w\rangle)^{2}-4 z_{1} z_{2} \bar{w}_{1} \bar{w}_{2}\right]^{3}} \quad\left(z, w \in D_{22}\right),
$$

where $\langle z, w\rangle:=z_{1} \bar{w}_{1}+z_{2} \bar{w}_{2}$, and also an explicit form of the Bergman metric for $D_{22}$ which we do not include here. 


\section{REFERENCES}

1. K. Azukawa and M. Suzuki, The Bergman metric on Thullen domain, Nagoya Math. J. 89 (1983), 1-11.

2. K. Azukawa, Bergman metric on a domain of Thullen type, Math. Rep. Toyama Univ. 7 (1984), 41-65.

3. E. Bedford and J. E. Fornaess, Biholomorphic maps of weakly pseudoconvex domains, Duke Math. J. 45 (1978), 711-719.

4. S. Bergman, Zur Theorie von pseudokonformen Abbildungen, Mat. Sb. 1 (43) (1936), 79-96.

5. D. Catlin, Invariant metrics on pseudoconvex domains, Several Complex Variables, Proceedings of the 1981 Hangzhou Conference, 1984, pp. 7-12.

6. K. Diederich, J. E. Fornaess, and G. Herbort, Boundary behavior of the Bergman metric, Proc. Sympos. Pure Math., vol. 41, Amer. Math. Soc., Providence, R. I., 1982, pp. 59-67.

7. I. Graham, Boundary behavior of the Carathéodory and Kobayashi metrics on strongly pseudoconvex domains in $\mathbf{C}^{n}$ with smooth boundary, Trans. Amer. Math. Soc. 207 (1975), 219-240.

8. R. Gunning and H. Rossi, Analytic functions of several complex variables, Prentice-Hall, Englewood Cliffs, N. J., 1965.

9. K. T. Hahn and P. Pflug, On a minimal complex norm that extends the real euclidean norm, Monatsh. Math. 105 (1988), 107-112.

10. S. Kobayashi, Hyperbolic manifolds and holomorphic mappings, Pure Appl. Math., Dekker, 1970.

11. N. Kritikos, Über analytische Abbildungen einer Klasse von vierdimensionalen Gebieten, Math. Ann. 99 (1928), 321-341.

12. L. Lempert, La métrique de Kobayashi et la représentation des domaines sur la boule, Bull. Soc. Math. France 109 (1981), 427-474.

13. P. Pflug, Glatte Holomorphiegebiete mit plurisubharmonischer innerer Randfunktion sind BanachStein, Ark. Mat. 14 (1976), 55-58.

14. M. Range, The Carathéodory metric and holomorphic maps on a class of weakly pseudoconvex domains, Pacific J. Math. 78 (1978), 173-189.

Department of Mathematics, Pennsylvania State University, University PARK, PENNSYLVANIA 16802

Departement der Mathematik, Universität OsnabruCK, Abteilung Vechta, D 2848 VECHTA, FEDERAL REPUBLIC OF GERMANY 